\title{
Increased MMP-9 and TIMP-1 in mouse neonatal brain and plasma and in human neonatal plasma after hypoxia- ischemia: a potential marker of neonatal encephalopathy
}

\author{
Nathalie Bednarek ${ }^{1-4}$, Pernilla Svedin ${ }^{5}$, Roselyne Garnotel ${ }^{6}$, Géraldine Favrais ${ }^{1-3}$, Gauthier Loron ${ }^{1-4}$, Leslie Schwendiman ${ }^{1-3}$, \\ Henrik Hagberg ${ }^{7}$, Patrice Morville ${ }^{4}$, Carina Mallard ${ }^{5}$ and Pierre Gressens ${ }^{1-3,7}$
}

\begin{abstract}
INTRODUCTION: To implement neuroprotective strategies in newborns, sensitive and specific biomarkers are needed for identifying those who are at risk for brain damage. We evaluated the effectiveness of matrix metalloproteinases (MMPs) and their naturally occurring tissue inhibitors of metalloproteinases (TIMPs) in predicting neonatal encephalopathy (NE) damage in newborns.
\end{abstract}

RESULTS: Plasma MMP-9 and TIMP-1 levels were upregulated as early as $1 \mathrm{~h}$ after the $\mathrm{HI}$ insult but not did not show such elevations after other types of injury (ibotenate-induced excitotoxicity, hypoxia, lipopolysaccharide-induced inflammation), and brain levels reflected this increase soon thereafter. We confirmed these results by carrying out plasma MMP-9 and TIMP-1 measurements in human newborns with NE. In these infants, protein levels of MMP-9 and TIMP-1 were found to be elevated during a short window up to $6 \mathrm{~h}$ after birth.

DISCUSSION: This feature is particularly useful in identifying newborns in need of neuroprotection. A second peak observed $72 \mathrm{~h}$ after birth is possibly related to the second phase of energy failure after a HI insult. Our data, although preliminary, support the use of MMP-9 and TIMP-1 as early biomarkers for the presence and extent of perinatal brain injury in human term newborns.

METHODS: We first used a mouse model of neonatal HI injury to explore mechanistic aspects such as the time course of these markers after the hypoxia-ischemia event, and the correlation between the levels of these candidate markers in brain and plasma.

$\mathbf{T}$ he major perinatal brain lesions associated with cerebral palsy and cognitive impairment are periventricular white matter damage and cortical-subcortical lesions, observed mainly in preterm and term infants, respectively $(1,2)$. A crucial step for the implementation of neuroprotective therapies is the rapid detection of neonates at risk. Despite this urgent need, no appropriate and easily detectable biomarkers for perinatal injury are currently available.
Matrix metalloproteinases (MMPs) and tissue inhibitors of metalloproteinases (TIMPs) are involved in multiple processes during development and in adulthood (3-5), including perinatal hypoxia-ischemia (HI) and white matter damage in human neonates. Recent studies in adult MMP-9 knockout mice have demonstrated the key role played by this metalloproteinase in the pathophysiology of traumatic and hypoxic-ischemic (HI) brain injury (6). Concordantly, MMP-9 has been shown to have deleterious effects in the immature brain after $\mathrm{HI}$ injury (7). Several of these studies demonstrated a significant increase in MMP-9 expression in the central nervous system within the first $24 \mathrm{~h}$ after the insult. MMP-9 also has long-term beneficial effects on neurovascular remodeling and behavioral recovery after stroke in adult rats $(8,9)$. In the adult rat central nervous system, elevated TIMP-1 expression levels may play a neuroprotective role after kainate-induced excitotoxic seizures (10), and gene transfer of TIMP- 1 and 2 protects against global cerebral ischemia in mice $(11,12)$. The fact that MMPs and TIMPs are secreted proteins that can easily be measured in bodily fluids including peripheral blood (13) highlights their potential as biomarkers for brain injury. Indeed, MMP-9 has already been evaluated as a biomarker for acute ischemic stroke in adult patients (14). However, their usefulness in neonates has not so far been demonstrated.

As a first step in the validation of MMPs and/or TIMPs as biomarkers for perinatal brain injury, we used a model of HI injury in postnatal day 9 (P9) mice (which mimics the gray matter lesions observed in human neonatal encephalopathy (NE)) (15), to study the correlation between plasma levels and brain levels of these molecules. We evaluated the specificity of these molecules for HI damage by measuring MMP and TIMP levels in two other conditions: ibotenate-induced excitotoxic lesions in P5 mice (which mimic periventricular white matter damage in preterm infants) (16), and lipopolysaccharide (LPS) injections (which mimic bacterial infection/inflammation in addition to sensitizing the brain to $\mathrm{HI}$ insults) (17). Over and above the mechanistic insights yielded by the use of animal models, we obtained information

${ }^{1}$ Inserm, Hôpital Robert Debré, Paris, France; ${ }^{2}$ Université Paris 7, Faculté de Médecine Denis Diderot, Paris, France; ${ }^{3}$ PremUP, Paris, France; ${ }^{4}$ Neonatalogy Department, Alix de Champagne, Reims, France; ${ }^{5}$ Göteborg University, Göteborg, Sweden; ${ }^{6}$ CNRS UMR 6237, Université de Reims Champagne-Ardennes, Reims, France; ${ }^{7}$ Institute for Reproductive and Developmental Biology, Imperial College, Hammersmith Campus, London, UK. Correspondence: Nathalie Bednarek (nbednarek@chu-reims.fr) 
regarding the potential of these biomarkers for human use by measuring MMP and TIMP levels in plasma collected within the first $6 \mathrm{~h}$ of life from infants with NE, as well as from healthy controls and from infants with infectious disease.

\section{RESULTS}

\section{Levels of MMPs and TIMPs in Plasma and Neocortex in HI Mice} Gelatinolytic activity of MMP-2 in the neocortex (Figure 1a,b) and plasma (Figure $2 \mathrm{a}-\mathrm{c}$ for $1 \mathrm{~h}$; other time points not shown) and MMP-2 protein levels (data not shown) were not affected by $\mathrm{HI}$ as compared to controls. Neocortical gelatinolytic activity (Figure 1a,b) and protein levels of MMP-9 (Figure 3a) were significantly increased at $24 \mathrm{~h}$ and 3 days after HI. Plasma MMP-9 protein levels were significantly increased at 1,3 , and $6 \mathrm{~h}$ and 3 days after HI, relative to controls (Figure $3 \mathbf{b}$ ).

As with MMP-9, neocortical TIMP-1 protein levels showed significant $\mathrm{HI}$-induced increases at $24 \mathrm{~h}$ and 3 days (Figure $3 \mathrm{c}$ ). Plasma TIMP-1 protein levels showed increases at even earlier time points, i.e., within the first few hours, and for as long as 3 days after the insult (Figure $3 \mathrm{~d}$ ). TIMP-2 protein levels could not be evaluated because of the lack of enzyme-linked immunosorbent assay kits sensitive enough for murine tissues.

\section{Levels of MMPs and TIMPs in Plasma and Neocortex of Mice With} Excitotoxic Lesions

Gelatinolytic activity and protein levels of MMP-2 (data not shown) and MMP-9 in the neocortices (Figure 3a) and plasma (Figures 2a,c and 3b) of P5 Swiss mice injected with ibotenate

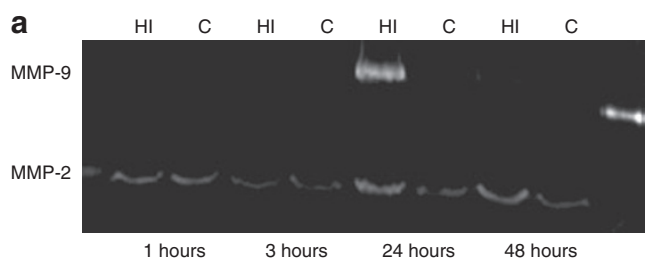

b

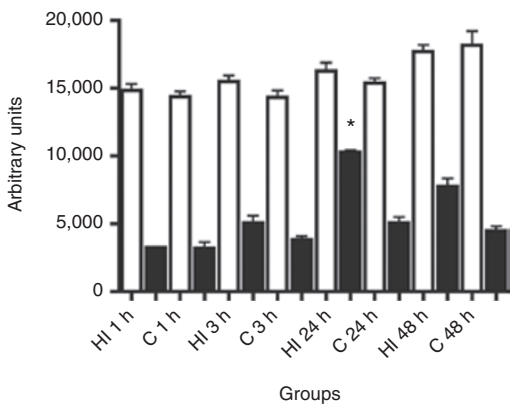

Figure 1. MMP-2/MMP-9 gelatinolytic activity in the neocortices of mice with $\mathrm{HI}$ brain injury. (a) Neocortical MMP-2 and MMP-9 gelatinolytic activity in $\mathrm{HI}$ mouse neonates killed 1, 3, 24, and $48 \mathrm{~h}$ after an $\mathrm{HI}$ insult, as compared to controls (C) (samples for $6 \mathrm{~h}$, and for 3, 4, and 5 days are not shown). (b) Quantitative zymography. White bars: MMP-2 gelatinolytic activity; black bars: MMP-9 gelatinolytic activity. The effect of $\mathrm{HI}$ on MMP-9 gelatinolytic activity was extremely significant $(P<0.001)$ at $24 \mathrm{~h}$. Similar results were observed at 3 days (data not shown). The effect of $\mathrm{HI}$ on MMP-2 levels was nonsignificant at all ages. ${ }^{*} P<0.001$; comparisons between $\mathrm{HI}$ groups and C; one-way ANOVA and Bonferroni post hoc test. $\mathrm{HI}$, hypoxia-ischemia; MMP, matrix metalloproteinase. were not different from the levels in controls at any time point. The gelatinolytic activity of neocortical MMP-9 after ibotenate injection in P9 C57BL/6 mice was also negligible at all time points studied, and plasma MMP-9 levels revealed no difference from those of controls (data not shown).

Neocortical TIMP-1 protein levels (Figure 3c) were briefly but significantly higher $24 \mathrm{~h}$ after ibotenate injection in P5 Swiss mice as compared to control mice. No increase was observed in the plasma protein levels (Figure 3d). At all other time points, TIMP-1 protein levels in ibotenate-treated and control mice were comparable.

Levels of MMPs and TIMPs in Plasma and Neocortices of hypoxic and LPS-exposed Mice

There was no increase in neocortical gelatinolytic activity or in levels of MMP-9 (Figure 3a), MMP-2 (data not shown), or TIMP-1 (Figure 3c) in the affected hemisphere in hypoxic

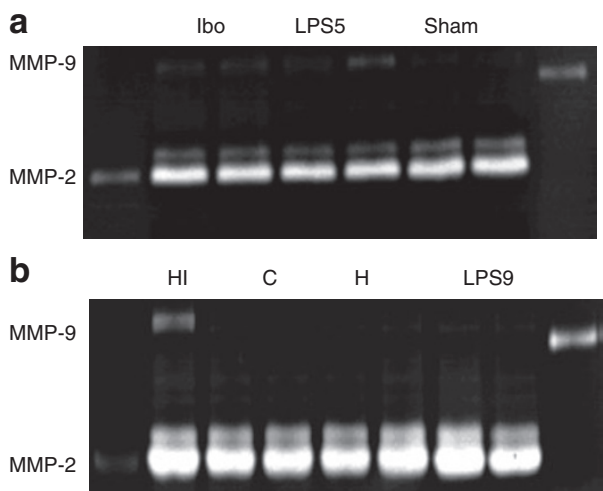

C

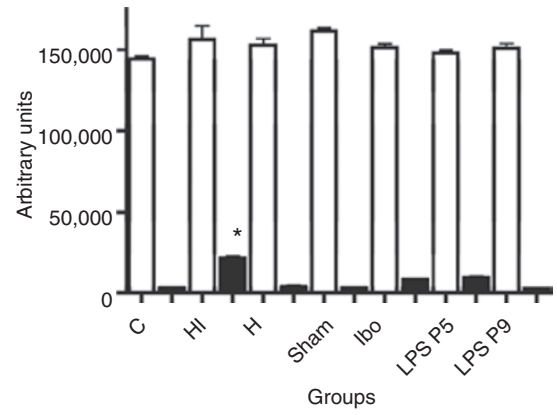

Figure 2. MMP-9 and TIMP-1 levels in brains and plasma of mice with hypoxic-ischemic $(\mathrm{HI})$, hypoxia alone $(\mathrm{H})$, and excitotoxic brain injuries. Protein levels in the $(\mathbf{a}, \mathbf{c})$ neocortices and $(\mathbf{b}, \mathbf{d})$ plasma of neonatal mice killed at 1, 3, 6, 24, and $48 \mathrm{~h}$, and at 3,4, and 5 days after the insult, in $\mathrm{HI}$ (white bars), H (light gray bars), ibotenate-injected (Ibo; dark gray bars), and controls ( $C$; black bars). Bars represent mean concentrations \pm SEM. ${ }^{*} P<0.001 ;{ }^{* *} P<0.01 ; \S P<0.05$; two-way analysis of variance with Bonferroni post hoc test. a,b: MMP-9 concentrations in (a) the neocortex and (b) plasma at various time points. The effect of HI was extremely significant at $24 \mathrm{~h}(P<0.001)$ and 3 days $(P<0.01)$ in the neocortex, and was significant at $1 \mathrm{~h}(P<0.001), 3 \mathrm{~h}(P<0.01), 6 \mathrm{~h}(P<0.05)$, and 3 days $(P<0.001)$ in plasma, relative to $C$ mice. Neither $\mathrm{H}$ nor Ibo affected MMP-9 protein levels. $\mathbf{c}, \mathbf{d}$ : TIMP-1 concentrations in (c) the neocortex and (d) plasma at various time points. The effect of $\mathrm{HI}$ was extremely significant at $24 \mathrm{~h}(P<0.001)$ and 3 days $(P<0.01)$ in the neocortex, and from $1 \mathrm{~h}$ up to 3 days $(P<0.001)$ in plasma, relative to $C$ mice. The effect of an excitotoxic (Ibo) injury on neocortical TIMP-1 levels was extremely significant at $24 \mathrm{~h}$ only $(P<0.001)$, whereas plasma levels were unchanged. $H$ did not affect TIMP-1 protein levels. MMP, matrix metalloproteinase; TIMP, tissue inhibitor of metalloproteinase. 

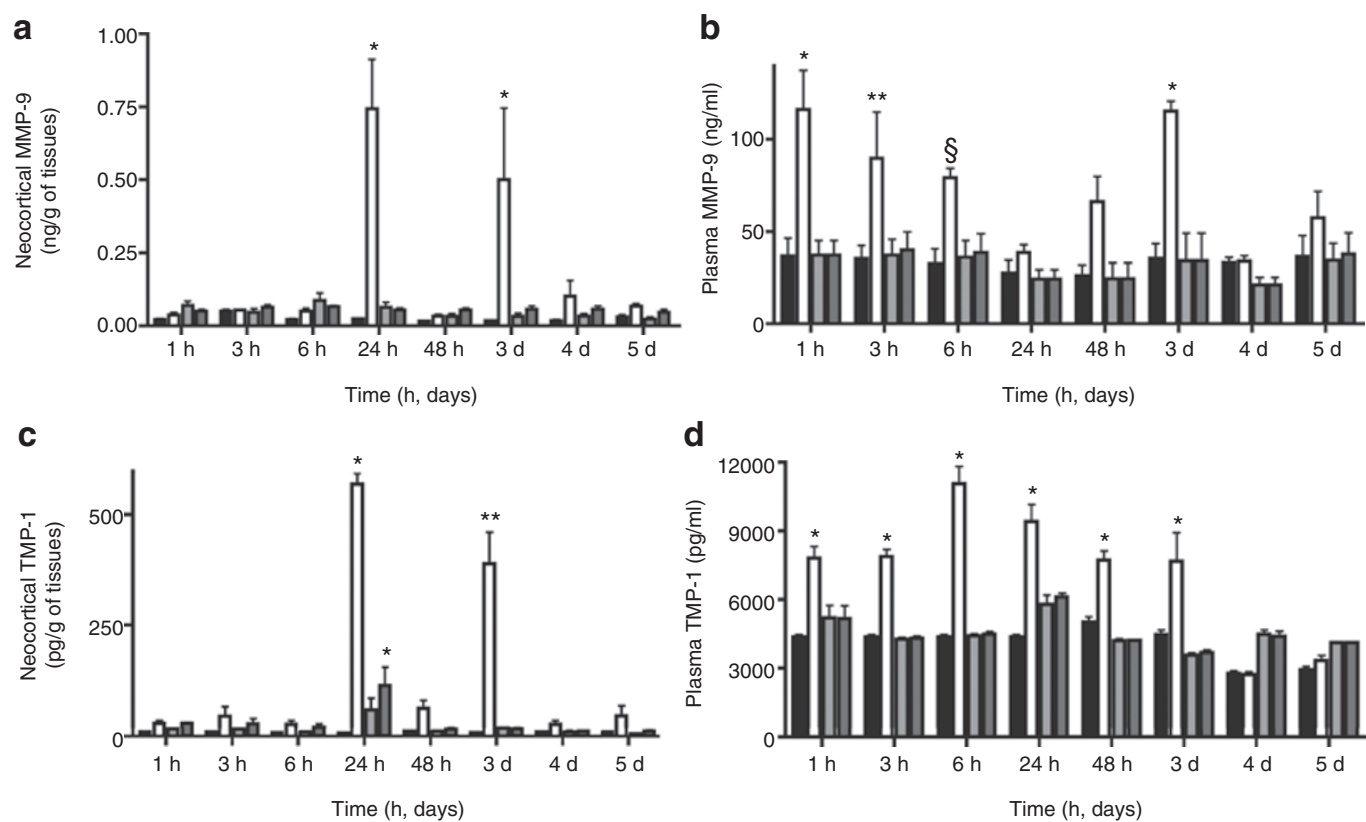

Figure 3. MMP-2 and MMP-9 levels in mice with excitotoxic, LPS-induced, $\mathrm{HI}$, or $\mathrm{H}$ brain injuries, and their controls. (a) Representative gelatinolytic activity in the plasma of mouse pups sacrificed $1 \mathrm{~h}$ after an excitotoxic (ibotenate-injected (Ibo)) insult at P5, and after LPS-induced inflammation at the same age (LPS 5), when compared to sham-operated mice at P9. None of the treatment groups showed any change in MMP-9 or MMP-2 activity when compared with controls (sham) at this or any other time point. (b) Representative gelatinolytic activity in the plasma of mouse pups sacrificed $1 \mathrm{~h}$ after $\mathrm{HI}$, $\mathrm{H}$ alone, or LPS-induced inflammation at P9, as compared to nonoperated controls (C). Although MMP-9 activity was upregulated at $1 \mathrm{~h}$ in the $\mathrm{HI}$ group, as well as at later time points (see Figure 1), neither MMP-9 nor MMP-2 activity was affected in any other group or time point studied. (c) Quantitative zymography in plasma $1 \mathrm{~h}$ after HI (P9), H (P9), Ibo (P5), and LPS (P5 and at P9), and in nonoperated C (P9) and sham-operated animals (P9). White bars: MMP-2 gelatinolytic activity; black bars: MMP-9 gelatinolytic activity. Only the HI group showed a significant increase in MMP-9 activity when compared to C. MMP-2 activity did not change in any group or time. ${ }^{*} P<0.001$; one-way analysis of variance with Bonferroni post hoc test. $\mathrm{H}$, hypoxia alone; $\mathrm{HI}$, hypoxia-ischemia; LPS, lipopolysaccharide; MMP, matrix metalloproteinase; P, postnatal day.

animals $(\mathrm{H})$ as compared with those in controls. Plasma MMP-9 and MMP-2 gelatinolytic activities were not higher in $\mathrm{H}$, sham-operated, or LPS-exposed animals (P9 or P5) as compared to controls, at any of the time points studied (Figure 2a-c show data for time point $1 \mathrm{~h}$; data for other time points not shown).

\section{Levels of MMPs and TIMPs in Human Plasma Samples} Controlgroup. MMP-2, TIMP-1, and TIMP-2 were easily detected at all ages using enzyme-linked immunosorbent assay; age and gender did not affect the results (data not shown). Mean values, SD, and range for MMP-2, MMP-9, TIMP-1, and TIMP-2 levels in plasma are shown in Table 1. Plasma MMP-9 levels were significantly lower in 25- to 27 -week-old infants than in those of higher gestational age $(P \leq 0.05$; see Table 1$)$. For this reason, and to exclude any effect of extreme prematurity not pertinent to this study, only data from preterm infants of gestational age $\geq 34$ weeks were analyzed as controls (Table 1).

NE group. (Table 2) MMP-2 and TIMP-2 levels were not influenced by pathological conditions (data not shown). However, MMP-9 levels were significantly increased in the NE group (mean \pm SD: $342 \pm 312 \mathrm{ng}$; median: $189 \mathrm{ng}$; range $=6-1,313 \mathrm{ng}$ ) as compared to controls; the wide range of values reflected the fact that the group included Sarnat stage 1 (S1) infants (not different from controls) along with S2 and S3 infants (Figure 4a).
No differences in MMP-9 levels were observed between NE patients with a favorable outcome and those with an unfavorable outcome (Figure 4a). TIMP-1 levels were significantly higher in NE patients than in controls (Figure $4 \mathrm{~b})(570 \pm 382 \mathrm{ng}$; range $=107-1,551 \mathrm{ng}$ ), mainly because of the presence of S2 infants in the group (Figure $4 \mathrm{~b}$ ); also, these levels were higher in NE patients with a favorable outcome than in those with an unfavorable outcome (Figure 4b).

Infectious group. (Table 3) Systemic infection did not influence MMP-2 (data not shown), MMP-9 (Figure 4a), TIMP-1 (Figure 4b), or TIMP-2 protein levels (data not shown).

\section{DISCUSSION}

This study demonstrates not only that MMP-9 and TIMP- 1 levels are elevated in both NE infants and in neonatal mice with $\mathrm{HI}$ brain injury, but also, remarkably, that these changes are measurable in plasma at an early time point after the insult. These results are corroborated by early elevated MMP- 9 and TIMP-1 levels in the neocortices of HI mice. Of interest, in human infants, MMP-9 plasma levels were correlated with the severity of NE, whereas TIMP-1 plasma levels were predictive of long-term sequelae. The significant increase in plasma MMP-9 levels within the first $6 \mathrm{~h}$ after birth is of critical importance in selecting newborns at high risk for neurological damage, or those most likely to benefit from neuroprotection. MMP-9 levels after $6 \mathrm{~h}$ after birth may be less informative and may include false-negative results. 
Table 1. Characteristics of infants without NE

\begin{tabular}{|c|c|c|c|c|c|}
\hline Patient & Sex $^{a}$ & $G^{b}$ & $\begin{array}{l}\text { MMP-9 } \\
\text { (ng/ml) }\end{array}$ & $\begin{array}{l}\text { TIMP-1 } \\
\text { (ng/ml) }\end{array}$ & $\begin{array}{c}\text { Timing of blood } \\
\text { sampling }\end{array}$ \\
\hline 1 & $\mathrm{~F}$ & 34 & 53 & 336 & 3 \\
\hline 2 & $\mathrm{~F}$ & 34 & 8 & 318 & 3.5 \\
\hline 3 & $\mathrm{~F}$ & 34 & 26 & 398 & 2 \\
\hline 4 & $M$ & 34 & 56 & 311 & 1 \\
\hline 5 & $M$ & 34 & 87 & 338 & 6 \\
\hline 6 & $\mathrm{~F}$ & 35 & 64 & 336 & 3 \\
\hline 7 & $\mathrm{~F}$ & 35 & 28 & 400 & 5 \\
\hline 8 & $M$ & 35 & 29 & 566 & 6 \\
\hline 9 & $M$ & 35 & 39 & 359 & 5.5 \\
\hline 10 & $M$ & 35 & 31 & 404 & 3 \\
\hline 11 & $\mathrm{~F}$ & 36 & 30 & 375 & 5 \\
\hline 12 & $\mathrm{~F}$ & 36 & 24 & 402 & 2 \\
\hline 13 & $\mathrm{~F}$ & 36 & 57 & 445 & 1 \\
\hline 14 & $\mathrm{~F}$ & 36 & 22 & 301 & 5 \\
\hline 15 & $M$ & 36 & 23 & 370 & 3 \\
\hline 16 & $\mathrm{~F}$ & 37 & 84 & 422 & 5 \\
\hline 17 & $\mathrm{~F}$ & 37 & 99 & 203 & 6 \\
\hline 18 & $M$ & 37 & 24 & 237 & 3 \\
\hline 19 & $M$ & 37 & 33 & 225 & 1 \\
\hline 20 & $M$ & 37 & 34 & 344 & 5 \\
\hline 21 & $\mathrm{~F}$ & 38 & 35 & 228 & 3 \\
\hline 22 & $M$ & 38 & 36 & 315 & 2 \\
\hline 23 & $M$ & 38 & 29 & 401 & 1 \\
\hline 24 & $M$ & 38 & 34 & 173 & 3 \\
\hline 25 & $\mathrm{~F}$ & 38 & 33 & 300 & 4 \\
\hline 26 & $\mathrm{~F}$ & 39 & 25 & 465 & 6 \\
\hline 27 & $\mathrm{~F}$ & 39 & 52 & 214 & 3 \\
\hline 28 & $\mathrm{~F}$ & 39 & 53 & 213 & 1 \\
\hline 29 & $M$ & 39 & 35 & 254 & 3 \\
\hline 30 & $M$ & 39 & 36 & 231 & 5 \\
\hline 31 & $\mathrm{~F}$ & 40 & 28 & 393 & 1 \\
\hline 32 & $\mathrm{~F}$ & 40 & 35 & 252 & 6 \\
\hline 33 & $\mathrm{~F}$ & 40 & 12 & 197 & 4 \\
\hline 34 & $M$ & 40 & 16 & 223 & 1 \\
\hline 35 & $M$ & 40 & 41 & 289 & 3 \\
\hline 36 & $\mathrm{~F}$ & 41 & 40 & 259 & 2 \\
\hline 37 & $\mathrm{~F}$ & 41 & 17 & 335 & 1 \\
\hline 38 & $\mathrm{~F}$ & 41 & 29 & 278 & 5 \\
\hline 39 & $M$ & 41 & 33 & 242 & 3 \\
\hline 40 & $\mathrm{~F}$ & 34 & 112 & 497 & 1 \\
\hline 41 & $\mathrm{~F}$ & 34 & 130 & 420 & 1 \\
\hline 42 & $M$ & 34 & 43 & 204 & 3 \\
\hline 43 & $M$ & 34 & 14 & 376 & 1 \\
\hline 44 & $\mathrm{~F}$ & 35 & 15 & 490 & 2 \\
\hline 45 & $\mathrm{~F}$ & 35 & 16 & 366 & 4.5 \\
\hline
\end{tabular}

Table 1. Continued

\begin{tabular}{|c|c|c|c|c|c|}
\hline Patient & $\operatorname{Sex}^{\mathrm{a}}$ & $\mathrm{GA}^{\mathrm{b}}$ & $\begin{array}{l}\text { MMP-9 } \\
\text { (ng/ml) }\end{array}$ & $\begin{array}{l}\text { TIMP-1 } \\
\text { (ng/ml) }\end{array}$ & $\begin{array}{l}\text { Timing of blood } \\
\text { sampling }\end{array}$ \\
\hline 46 & $M$ & 35 & 52 & 378 & 5 \\
\hline 47 & $M$ & 35 & 86 & 562 & 5.5 \\
\hline 48 & $M$ & 35 & 20 & 194 & 3 \\
\hline 49 & $M$ & 35 & 104 & 243 & 1 \\
\hline 50 & $\mathrm{~F}$ & 36 & 20 & 275 & 3.5 \\
\hline 51 & $\mathrm{~F}$ & 36 & 10 & 231 & 5 \\
\hline 52 & $\mathrm{~F}$ & 36 & 64 & 145 & 3 \\
\hline 53 & $M$ & 36 & 10 & 168 & 1 \\
\hline 54 & $\mathrm{~F}$ & 37 & 12 & 204 & 2 \\
\hline 55 & $\mathrm{~F}$ & 37 & 59 & 168 & 2 \\
\hline 56 & $M$ & 37 & 34 & 340 & 1 \\
\hline 57 & $M$ & 37 & 13 & 196 & 5.5 \\
\hline 58 & $\mathrm{~F}$ & 38 & 52 & 184 & 4 \\
\hline 59 & $\mathrm{~F}$ & 38 & 11 & 190 & 1 \\
\hline 60 & $M$ & 38 & 31 & 185 & 2 \\
\hline 61 & $M$ & 38 & 10 & 171 & 2 \\
\hline 62 & $\mathrm{~F}$ & 39 & 100 & 353 & 1 \\
\hline 63 & $\mathrm{~F}$ & 39 & 10 & 340 & 3 \\
\hline 64 & $M$ & 39 & 22 & 196 & 4 \\
\hline 65 & $M$ & 39 & 14 & 171 & 1 \\
\hline 66 & $\mathrm{~F}$ & 40 & 112 & 234 & 3 \\
\hline 67 & $\mathrm{~F}$ & 40 & 30 & 257 & 1 \\
\hline 68 & $M$ & 40 & 24 & 227 & 2.5 \\
\hline 69 & $M$ & 40 & 23 & 181 & 2 \\
\hline 70 & $\mathrm{~F}$ & 41 & 23 & 243 & 1 \\
\hline 71 & $M$ & 41 & 60 & 378 & 3 \\
\hline 72 & $M$ & 41 & 76 & 254 & 5 \\
\hline
\end{tabular}

Numbers 1-39: control patients, numbers 40-72: infected patients.

MMP, matrix metalloproteinase; NE, neonatal encephalopathy; TIMP, tissue inhibitor of metalloproteinase. ${ }^{\text {SS }}$ ex: $\mathrm{M}=$ male, $\mathrm{F}=$ female. ${ }^{\mathrm{b}} \mathrm{GA}$ : gestational age in weeks. ${ }^{\mathrm{c}}$ Hours after birth.

Of note, none of the other pathological conditions studiedexcitotoxic lesions, hypoxia, or LPS-induced inflammationaffected MMP-9 and TIMP-1 protein levels in mouse brain or plasma, although the duration of the experiment was sufficient for MMP-9 and TIMP-1 activation in these groups; in contrast, MMP-9 levels in HI mice increased as early as $1 \mathrm{~h}$ after the insult. Excitotoxic and inflammatory processes are involved in both the early phase of brain ischemia and the regulation of MMP expression. However, one cannot rule out the possibility that the severity of tissue damage is a factor contributing to the increase in MMP-9 and TIMP-1 expression in HI mice, given that brain lesions are known to be more severe from a histological viewpoint in the HI model than in the excitotoxin models $(7,18)$.

Similarly, although we used two different strains of mice (Swiss for ibotenate and C57Bl/6 for HI), it is unlikely that differences in the strain used greatly influenced our results, 
Table 2. Characteristics of infants with NE

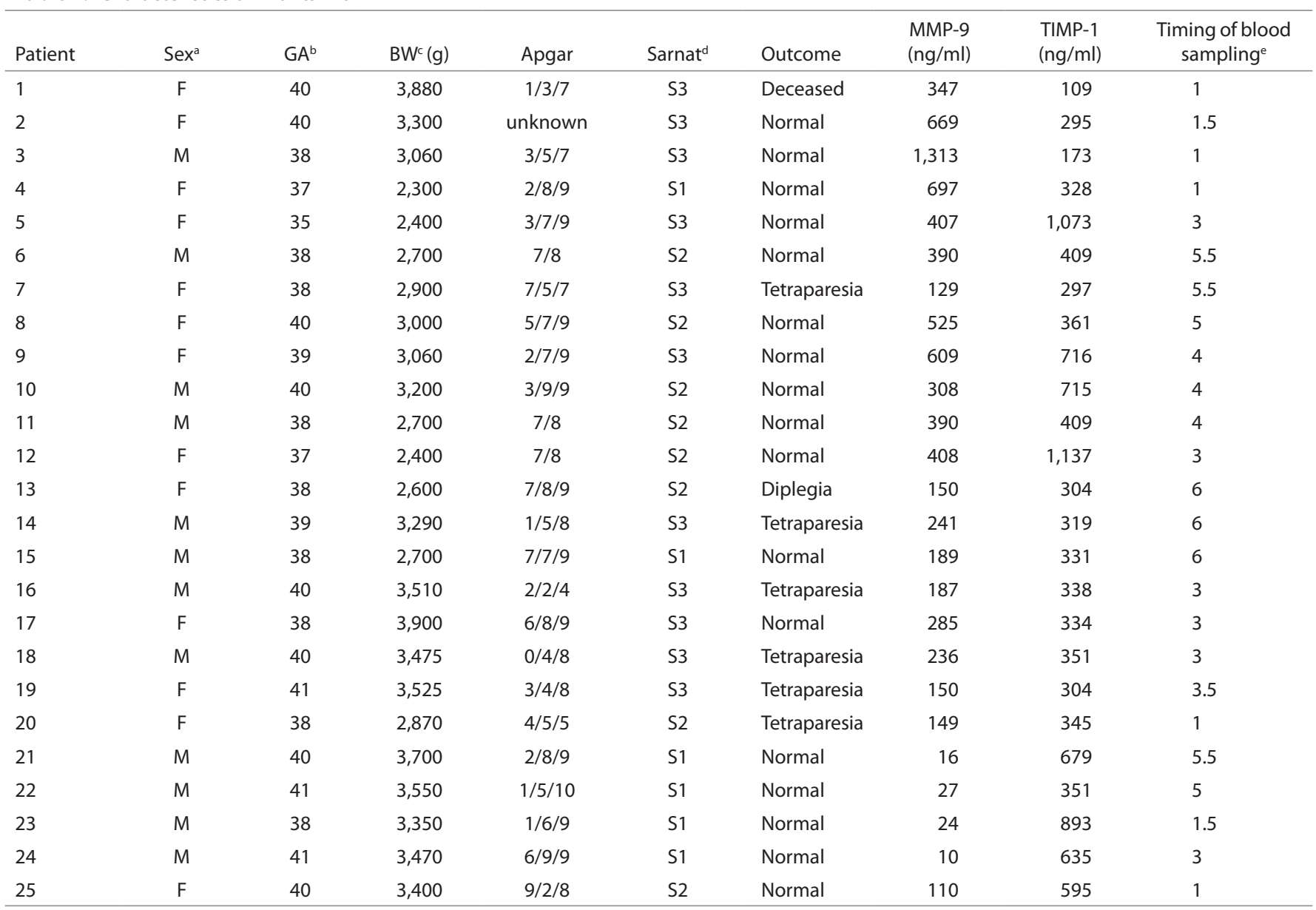

MMP, matrix metalloproteinase; NE, neonatal encephalopathy; TIMP, tissue inhibitor of metalloproteinase.

aSex: $M=$ male, $F=$ female. ${ }^{b} G A$ : gestational age in weeks. 'BW: birth weight in grams. ${ }^{~}$ Sarnat stage 1, 2, 3: S1, S2, S3. eHours after birth.

because we have previously shown that the ontogeny of MMP2, MMP-9, TIMP-1, and TIMP-2 is strain-independent (19). Differences in the ages of the two sets of mice (P9 for HI and P5 for ibotenate) can also be eliminated as a factor, because ibotenate injected at age P9 in C57BL/6 mice did not increase plasma MMP-9 levels. In fact, under physiological conditions, MMP-9 is undetectable in the postnatal mouse brain (19). Of interest, hypoxia by itself did not affect levels of MMP-9 and TIMP-1 in the brain, suggesting that ischemia is the key contributor to the observed increases in MMP-9 and TIMP-1 expressions.

In the study in human newborns also, those with a systemic infection did not display any increase in MMP-9 or TIMP-1 levels relative to the controls, suggesting that systemic infection associated with neonatal NE does not interfere with the interpretation of plasma MMP-9 and TIMP-1 measurements, unlike other biomarkers such as lactates or cytokines that are also upregulated (20). Another advantage of measuring MMP-9 and TIMP-1 levels in plasma is the relative ease of access when compared with the collection of cerebrospinal fluid or urine samples that are generally required for other prognostic biomarkers.

The levels of MMP-9 and TIMP-1 in the neocortices and plasma of mice and in the plasma of human neonates are closely matched over time, suggesting that MMP-9 activity is tightly controlled by its endogenous inhibitor, TIMP-1. This balance between the two molecules is thought to reduce brain damage in $\mathrm{HI}$ events by protecting the blood-brain barrier (7). MMP inhibition provides neuroprotection against $\mathrm{HI}$ in the developing brain (21). A neuroprotective role for TIMP-1 in excitotoxic damage and ischemia has been previously proposed $(10,11,22)$. In addition, TIMP-1 levels have recently been shown to be correlated with stroke outcome in adult humans (23).

When comparing the profiles of MMP-9 and TIMP-1 over time in the neocortex and plasma, it appears that the levels of both molecules increase in the plasma before they increase in the neocortex ( $1 \mathrm{~h}$ vs. $24 \mathrm{~h}$ after the insult) and return to basal values earlier in plasma than in the neocortex ( 3 days vs. 5 days after the insult), suggesting that circulating MMP-9 and TIMP-1 do not originate in the brain. Although our data do not allow us to discriminate between peripheral origin and diffusion from the lesion site, one could speculate that early increases in plasma MMP-9 and TIMP-1 have a systemic source related to multiorgan injury after moderate/severe HI. The release of MMP-9 by peripheral blood leukocytes, known to be activated in neonatal asphyxia, could contribute to this elevation (24). The second plasma peak at $24 \mathrm{~h}$ in mice and human infants could be 

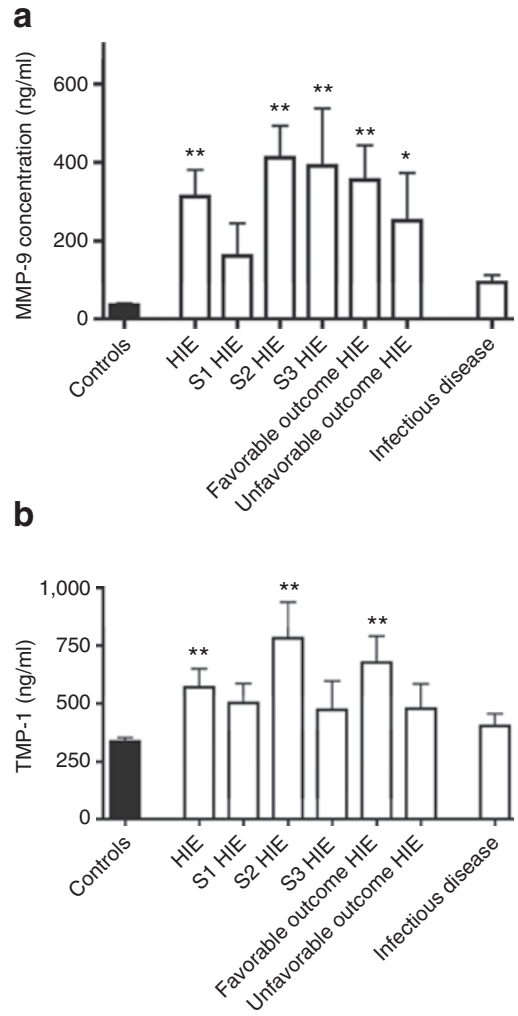

Figure 4. MMP- 9 and TIMP- 1 plasma levels in $<6$ h-old newborns with NE. Bars represent mean protein concentration \pm SEM. ${ }^{*} P<0.05$; ${ }^{*} P<0.01$; patient groups (white bars) vs. controls (black bar); analysis of variance and Bonferroni's multiple comparison test. (a) MMP-9 protein levels in the plasma of control (black bar), NE, NE differentiated according to Sarnat stage (S1, S2, S3) or according to favorable/unfavorable outcome following NE, and infected infants. Apart from the S1 group, MMP-9 levels in the plasma of all NE infants were significantly higher than in controls, regardless of Sarnat stage or outcome. (b) TIMP-1 protein levels in the plasma of control (black bar), NE, NE differentiated according to Sarnat stage (S1, S2, S3), or according to favorable or unfavorable outcome following NE, and infected infants. TIMP-1 levels were significantly higher in the plasma for all NE infants and those with S2 NE, as well as those with a favorable outcome. MMP, matrix metalloproteinase; TIMP, tissue inhibitor of metalloproteinase; NE, neonatal encephalopathy.

Table 3. MMP-2, -9 and TIMP-1, -2 normative values in the neonatal period

\begin{tabular}{lcc}
\hline & Mean value $(\mathrm{ng} / \mathrm{ml}) \pm \mathrm{SD}$ & Range \\
\hline MMP-2 & $2,461 \pm 610$ & $2,181-3,965$ \\
MMP-9 & $10.6 \pm 9.9^{\mathrm{a}}, 33 \pm 19.8^{\mathrm{b}}$ & $0-27^{\mathrm{a}}, 0-80^{\mathrm{b}}$ \\
TIMP-1 & $337.6 \pm 106.2$ & $157-689$ \\
TIMP-2 & $539 \pm 610$ & $209-954$ \\
\hline
\end{tabular}

Values for infants.

MMP, matrix metalloproteinase; TIMP, tissue inhibitor of metalloproteinase.

a 25-27 gestational weeks of age. ${ }^{b}>27$ gestational weeks of age.

the result of passive diffusion of MMP-9 and TIMP-1 produced in the brain, possibly by the death of neural cells as in the case of global ischemic injury (9). Alternatively, blood-brain barrier breakdown and the inflammatory response, involved early in focal injury, could lead to an increase not only in MMP-9 but also in MMP-2, MMP-3, and TIMP-3. However, reports regarding MMP-2 are often contradictory $(4,22,25,26)$, and we therefore preferred to focus on the more reliable marker, MMP-9 (9). The second peak of MMP-9 in the brain might also reflect the secondary phase of energy failure induced by HI (27). A second but considerably delayed peak of MMP-9 activity in the brain has also been seen by others (26).

Despite their uncertain origin, circulating MMP-9 and TIMP-1 levels are potential biomarkers of NE severity. The levels and significance of MMP-9 and MMP-2 have been previously evaluated in stroke in adult patients $(14,28)$. In adults, high plasma MMP-9 levels have been associated with the occurrence of early hemorrhagic complications after thrombolysis (28) and early deterioration during cerebral ischemia (29). However, to our knowledge, only one study has explored plasma levels of MMP-9 in perinatal asphyxia: MMP-9 and TIMP-1 levels measured on the day of birth in asphyxiated infants with sequelae were significantly higher than in infants with a favorable outcome or in control patients (30). The discrepancy between the results of that study and ours could be attributable to the low number of patients in the former, or the timing of blood sampling in that study, which was reported as occurring on the day of birth, but no details were mentioned. Although NE infants with an S1 in our study were not significantly different from controls in terms of MMP-9 plasma levels, those with higher Sarnat stages had significantly higher MMP-9 levels, in keeping with a correlation between levels of this protein and severity of brain damage. In contrast, plasma TIMP-1 levels showed a different pattern, being higher in S2 infants than in S1 or S3 infants. Although it is easy to dismiss low TIMP-1 levels in $\mathrm{S} 1$ infants as reflecting a lack of severe lesions, as suggested by their low MMP-9 levels, it could well be that the neuroprotective effects of TIMP-1 in the S2 and S3 groups are responsible for a more favorable outcome in the former, and vice versa.

Remarkably, plasma MMP-9 levels in the NE infants in our study were higher $(441.7 \pm 91.5 \mathrm{ng} / \mathrm{ml})$ than those observed in adult stroke patients $(147.1 \pm 118.6 \mathrm{ng} / \mathrm{ml})(30)$. One possible explanation is that the mechanism underlying perinatal $\mathrm{HI}$ events is associated with more global effects on different organs (e.g., umbilical cord compression/placental abruption) than is the ischemic mechanism (i.e., blood clot), which is restricted to the brain in adult stroke.

This study provides preliminary evidence that MMP-9 and TIMP-1 could act as early biomarkers of brain damage in NE, and offers new avenues for research that could benefit human full-term newborns. However, before the use of these proteins as biomarkers can be validated, more steps are required: (i) identifying their initial source of secretion, (ii) understanding the etiology of the biphasic secretion of MMP-9, and (iii) evaluating their specificity and sensitivity and correlating their concentration levels with the degree and type of brain damage in humans and in animal models.

\section{METHODS}

The study in human newborns was approved by the Reims Hospital ethics committee. Written informed consent was obtained from parents/ legal representatives of all patients enrolled in this study. Experimental protocols in animals were approved by the Institutional Animal Care 
and Use Committee of Robert Debre Hospital (Paris). C57Bl/6 and Swiss mice were used in the experiments.

\section{Subjects}

\section{Mouse neonates}

HI and hypoxic $(H)$ brain injury. An HI insult was induced at age P9 in C57Bl/6 mice by unilateral ligation of the left carotid followed by induced hypoxia ( $10 \%$ oxygen in nitrogen, humidified, for $50 \mathrm{~min}$ ) as previously described $(7,15)$. The neonatal mice were killed at 1,3 , 6,24 , and $48 \mathrm{~h}$ and 3,4 and 5 days after HI. The left hemisphere of the brain was analyzed to determine the extent of HI lesions. An insult involving hypoxia alone $(\mathrm{H})$ was induced at age $\mathrm{P} 9$ in a second group of $\mathrm{C} 57 \mathrm{Bl} / 6$ mice, using the same procedure, except for carotid ligation. The control group was composed of nonoperated neonatal mice that were also killed at the same time points. Another group consisted of sham-operated neonates (shams) subjected to cervical incision alone, and killed at $1,3,6$, and $24 \mathrm{~h}$.

Excitotoxic brain injury. Intracerebral injection of $10 \mu \mathrm{g}$ ibotenate (Sigma, St Louis, MO) was performed, as previously described, in P5 Swiss mice (16) and in P9 C57Bl/6 mice for making comparisons with data from $\mathrm{HI}$ animals. The neonatal mice were killed at the same time points as were those in the HI group. In determining protein levels in the brain, the brain hemisphere contralateral to the one receiving the ibotenate injection was used as the control.

Inflammation/infection group. An intraperitoneal injection of LPS $(0.3 \mathrm{mg} / \mathrm{kg})$ was administered at ages $\mathrm{P} 5$ and $\mathrm{P} 9$ to $\mathrm{C} 57 \mathrm{Bl} / 6$ mice, as previously described (17), to mimic the effects of systemic infection. The animals were killed at 1, 3, 6, and $24 \mathrm{~h}$ after injection, as in the sham-operated group.

Plasma and brain samples. Immediately before killing the mice, arterial blood was obtained from all of them and centrifuged at $3,000 \mathrm{~g}$. Plasma was obtained from additional animals and pooled (three animals per sample, five samples per experimental group), immediately frozen, and stored at $-80^{\circ} \mathrm{C}$. For neocortex samples, five animals were studied at each age and time point. Neocortices of $\mathrm{HI}, \mathrm{H}$, and control mice were rinsed in RPMI medium, immediately frozen, and stored at $-80^{\circ} \mathrm{C}$. The neocortices were analyzed individually.

Human neonates. Blood samples were collected prospectively within the first $6 \mathrm{~h}$ after birth from all of the newborns (gestational age 25-41 weeks) admitted to the Reims Hospital Neonatal Intensive Care Unit between May 2005 and May 2008. The samples were frozen immediately and stored at $-80^{\circ} \mathrm{C}$. The samples were processed by investigators who were blinded as to the identity of the subjects, and the diagnosis was revealed only after assay results were obtained.

Patients in the control group were those who showed no evidence of neonatal infectious disease and did not fulfill the criteria for NE at birth. In addition, they displayed normal neurological development (Denver scale) and normal growth parameters (follow-up from 2 to 5 years).

Diagnosis of NE was carried out in term infants (gestational age $>37$ weeks) displaying clinical signs of encephalopathy; these were classified on the basis of clinical severity, as described by Sarnat (S1-S3) (31). In addition, the infants selected showed supporting evidence of fetal distress (history of an acute event, cord $\mathrm{pH} \leq 7.1$, or base deficit $\geq 16 \mathrm{mEq} / \mathrm{l}$ ) and neonatal distress (Apgar score $\leq 5$ at $10 \mathrm{~min}$, blood gas $\mathrm{pH}$ within the first hour of life $\leq 7.1$ and/or base deficit $>16 \mathrm{mmol} / \mathrm{l}$, or the continued need for ventilation initiated at birth). The clinical characteristics of these patients are summarized in Table 2.

Infection was defined as any positive test on blood/cerebrospinal fluid culture or peripheral gastric, ear or anal samples for bacterial presence or for inflammatory markers (C-reactive protein $>10 \mathrm{mg} / \mathrm{l}$ and/or procalcitonin $>2 \mu \mathrm{g} / \mathrm{l}$ ), and/or an absolute neutrophil count $<1,500$.

\section{Measurement of MMPs and TIMPs}

Gelatin zymography. MMP-2 and MMP-9 activity levels were determined by gelatin zymography, as previously described (7). Neocortical $(100 \mu \mathrm{g}$ of protein) or plasma samples $(10 \mu \mathrm{l}$ per sample) were used for zymography. Human recombinant MMP-2 and MMP-9 (Millipore, Bedford, MA) were used as internal standards. Gelatinolytic activity was measured by means of automated image analysis (VilbertLourmat, Marne-la-Vallée, France).

Enzyme-linked immunosorbent assay. MMP-2 and MMP-9 levels and TIMP-1 and TIMP-2 levels were determined in duplicate using Quantikine ELISA kits (R\&D System, Lille, France) for human/mouse MMP-2, mouse pro-MMP-9, human TIMP-2, and mouse TIMP-1, as directed. Plasma samples were diluted three times before use; brain samples were used undiluted. The values obtained lay within the linear portion of the standard curve.

\section{Statistical Analyses}

Study group characteristics are described using the mean value and $\mathrm{SD}$ for continuous variables. One- and two-way analysis of variance were used to compare results (MMP-9/TIMP-1) among groups (sex, age group, presence of neonatal encephalopathy, Sarnat stage, favorable vs. unfavorable outcome, infants with infection vs. controls, ibotenate, and $\mathrm{HI}$ in mice), using a Bonferroni post hoc multiple comparison test where appropriate. A multiparameter correlation test was used for continuous variables (MMP-9, TIMP-1, gestational age, timing of blood sampling).

\section{ACKNOWLEDGMENTS}

We thank Jorge Gallego (INSERM U676), Ryan Colvin (St Louis Children's Hospital, Washington University, St Louis MO), and Damien Jolly (CHU Reims) for their help with the statistical analysis of the data; and Paul Toubas (State of University of New York, Downstate Medical Center, Brooklyn, New York), Pierre Desautels, and Amit Mathur (St Louis Children's Hospital, Washington University, St Louis MO) for critical reading of the manuscript.

\section{STATEMENT OF FINANCIAL SUPPORT}

This study was supported by a grant from the Seventh Framework Programme of the European Commission (grant agreement no. HEALTH-F22009-241778/neurobid), the American Memorial Inc (Boston, Paris), the INSERM, the Université Paris 7, the Swedish Research Council, the Assistance Publique, Hopitaux de Paris (APHP) (interface contract to Pierre Gressens), and the Leducq Foundation.

Disclosure: The authors have no financial or other conflicts of interest to declare.

\section{REFERENCES}

1. Okereafor A, Allsop J, Counsell SJ, et al. Patterns of brain injury in neonates exposed to perinatal sentinel events. Pediatrics 2008;121:906-14.

2. Bodensteiner JB, Johnsen SD. Magnetic resonance imaging (MRI) findings in children surviving extremely premature delivery and extremely low birthweight with cerebral palsy. J Child Neurol 2006;21:743-7.

3. Dzwonek J, Rylski M, Kaczmarek L. Matrix metalloproteinases and their endogenous inhibitors in neuronal physiology of the adult brain. FEBS Lett 2004;567:129-35.

4. Ranasinghe HS, Williams CE, Christophidis LJ, Mitchell MD, Fraser M, Scheepens A. Proteolytic activity during cortical development is distinct from that involved in hypoxic ischemic injury. Neuroscience 2009;158:732-44.

5. Agrawal SM, Lau L, Yong VW. MMPs in the central nervous system: where the good guys go bad. Semin Cell Dev Biol 2008;19:42-51.

6. Wang X, Jung J, Asahi M, et al. Effects of matrix metalloproteinase-9 gene knock-out on morphological and motor outcomes after traumatic brain injury. J Neurosci 2000;20:7037-42.

7. Svedin P, Hagberg H, Sävman K, Zhu C, Mallard C. Matrix metalloproteinase-9 gene knock-out protects the immature brain after cerebral hypoxia-ischemia. J Neurosci 2007;27:1511-8. 
8. Zhao BQ, Wang S, Kim HY, et al. Role of matrix metalloproteinases in delayed cortical responses after stroke. Nat Med 2006;12:441-5.

9. Cunningham LA, Wetzel M, Rosenberg GA. Multiple roles for MMPs and TIMPs in cerebral ischemia. Glia 2005;50:329-39.

10. Rivera S, Tremblay E, Timsit S, Canals O, Ben-Ari Y, Khrestchatisky M. Tissue inhibitor of metalloproteinases-1 (TIMP-1) is differentially induced in neurons and astrocytes after seizures: evidence for developmental, immediate early gene, and lesion response. J Neurosci 1997;17:4223-35.

11. Magnoni S, Baker A, Thomson S, et al. Neuroprotective effect of adenoviral-mediated gene transfer of TIMP-1 and -2 in ischemic brain injury. Gene Ther 2007;14:621-5.

12. Tejima E, Guo S, Murata Y, et al. Neuroprotective effects of overexpressing tissue inhibitor of metalloproteinase TIMP-1. J Neurotrauma 2009;26:1935-41.

13. Docherty AJ, O’Connell J, Crabbe T, Angal S, Murphy G. The matrix metalloproteinases and their natural inhibitors: prospects for treating degenerative tissue diseases. Trends Biotechnol 1992;10:200-7.

14. Montaner J, Alvarez-Sabín J, Molina C, et al. Matrix metalloproteinase expression after human cardioembolic stroke: temporal profile and relation to neurological impairment. Stroke 2001;32:1759-66.

15. Rice JE $3^{\text {rd }}$, Vannucci RC, Brierley JB. The influence of immaturity on hypoxic-ischemic brain damage in the rat. Ann Neurol 1981;9: 131-41.

16. Marret S, Mukendi R, Gadisseux JF, Gressens P, Evrard P. Effect of ibotenate on brain development: an excitotoxic mouse model of microgyria and posthypoxic-like lesions. J Neuropathol Exp Neurol 1995;54:358-70.

17. Eklind S, Mallard C, Leverin AL, et al. Bacterial endotoxin sensitizes the immature brain to hypoxic-ischaemic injury. Eur J Neurosci 2001;13:1101-6.

18. Medja F, Lelièvre V, Fontaine RH, et al. Thiorphan, a neutral endopeptidase inhibitor used for diarrhoea, is neuroprotective in newborn mice. Brain 2006;129(Pt 12):3209-23.

19. Bednarek N, Clément Y, Lelièvre V, et al. Ontogeny of MMPs and TIMPs in the murine neocortex. Pediatr Res 2009;65:296-300.

20. Volpe JJ. Neurology of the Newborn. Philadelphia, PA: Saunders, 2008:401-80.
21. Chen W, Hartman R, Ayer R, et al. Matrix metalloproteinases inhibition provides neuroprotection against hypoxia-ischemia in the developing brain. J Neurochem 2009;111:726-36.

22. Tan HK, Heywood D, Ralph GS, Bienemann A, Baker AH, Uney JB. Tissue inhibitor of metalloproteinase 1 inhibits excitotoxic cell death in neurons. Mol Cell Neurosci 2003;22:98-106.

23. Worthmann H, Tryc AB, Goldbecker A, et al. The temporal profile of inflammatory markers and mediators in blood after acute ischemic stroke differs depending on stroke outcome. Cerebrovasc Dis 2010;30:85-92.

24. Hasegawa K, Ichiyama T, Isumi H, Nakata M, Sase M, Furukawa S. NFkappaB activation in peripheral blood mononuclear cells in neonatal asphyxia. Clin Exp Immunol 2003;132:261-4.

25. Tong W, Chen W, Ostrowski RP, et al. Maternal hypoxia increases the activity of MMPs and decreases the expression of TIMPs in the brain of neonatal rats. Dev Neurobiol 2010;70:182-94.

26. Dragun P, Makarewicz D, Wójcik L, Ziemka-Nalecz M, Slomka M, Zalewska T. Matrix metaloproteinases activity during the evolution of hypoxic-ischemic brain damage in the immature rat. The effect of 1-methylnicotinamide (MNA). J Physiol Pharmacol 2008;59:441-55.

27. Lorek A, Takey Y, Cady EB, et al. Delayed ("secondary") cerebral energy failure after acute hypoxia-ischemia in the new born piglet: continuous 48 hours studies by phosphorus magnetic resonance spectroscopy. Ped Res 1994;36:699-706.

28. Montaner J, Molina CA, Monasterio J, et al. Matrix metalloproteinase-9 pretreatment level predicts intracranial hemorrhagic complications after thrombolysis in human stroke. Circulation 2003;107:598-603.

29. Lorenzl S, De Pasquale G, Segal AZ, Beal MF. Dysregulation of the levels of matrix metalloproteinases and tissue inhibitors of matrix metalloproteinases in the early phase of cerebral ischemia. Stroke 2003;34:e37-8; author reply e37-8.

30. Sunagawa S, Ichiyama T, Honda R, Fukunaga S, Maeba S, Furukawa S. Matrix metalloproteinase- 9 and tissue inhibitor of metalloproteinase-1 in perinatal asphyxia. Brain Dev 2009;31:588-93.

31. Sarnat HB, Sarnat MS. Neonatal encephalopathy following fetal distress. A clinical and electroencephalographic study. Arch Neurol 1976;33: 696-705. 